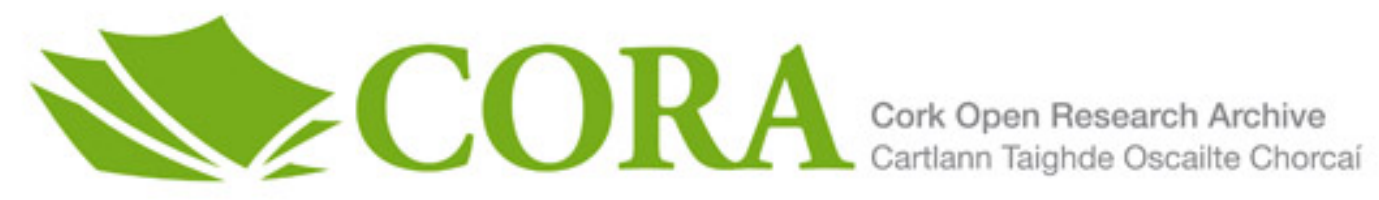

UCC Library and UCC researchers have made this item openly available. Please let us know how this has helped you. Thanks!

\begin{tabular}{|l|l|}
\hline Title & $\begin{array}{l}\text { Content-aware partial compression for textual big data analysis in } \\
\text { Hadoop }\end{array}$ \\
\hline Author(s) & Dong, Dapeng; Herbert, John \\
\hline Publication date & $2017-06-29$ \\
\hline Original citation & $\begin{array}{l}\text { Dong, D. and Herbert, J. (2017) 'Content-aware Partial Compression for } \\
\text { Textual Big Data Analysis in Hadoop', IEEE Transactions on Big Data, } \\
\text { In Press, doi: } 10.1109 / T B D A T A .2017 .2721431\end{array}$ \\
\hline Type of publication & Article (peer-reviewed) \\
\hline Link to publisher's \\
version & $\begin{array}{l}\text { http://dx.doi.org/10.1109/TBDATA.2017.2721431 } \\
\text { Access to the full text of the published version may require a } \\
\text { subscription. }\end{array}$ \\
\hline Rights & $\begin{array}{l}\text { C } 2017 \text { IEEE. Personal use of this material is permitted. Permission } \\
\text { from IEEE must be obtained for all other uses, in any current or } \\
\text { future media, including reprinting/republishing this material for } \\
\text { advertising or promotional purposes, creating new collective works, } \\
\text { for resale or redistribution to servers or lists, or reuse of any } \\
\text { copyrighted component of this work in other works. }\end{array}$ \\
\hline $\begin{array}{l}\text { Item downloaded } \\
\text { from }\end{array}$ & \begin{tabular}{l} 
http://hdl.handle.net/10468/5452 \\
\hline ing
\end{tabular} \\
\hline
\end{tabular}

Downloaded on 2020-07-06T14:31:29Z

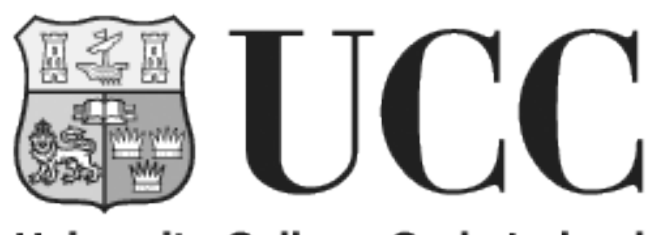




\title{
Content-aware Partial Compression for Textual Big Data Analysis in Hadoop
}

\author{
Dapeng Dong, Member, IEEE and John Herbert, Member, IEEE
}

\begin{abstract}
A substantial amount of information in companies and on the Internet is present in the form of text. The value of this semi-structured and unstructured data has been widely acknowledged, with consequent scientific and commercial exploitation. The ever-increasing data production, however, pushes data analytic platforms to their limit. Compression as an effective means to reduce data size has been employed by many emerging data analytic platforms, whom the main purpose of data compression is to save storage space and reduce data transmission cost over the network. Since general purpose compression methods endeavour to achieve higher compression ratios by leveraging data transformation techniques and contextual data, this context-dependency forces the access to the compressed data to be sequential. Processing such compressed data in parallel, such as desirable in a distributed environment, is extremely challenging. This work proposes techniques for more efficient textual big data analysis with an emphasis on content-aware compression schemes suitable for the Hadoop analytic platform. The compression schemes have been evaluated for a number of standard MapReduce analysis tasks using a collection of public and private real-world datasets. In comparison with existing solutions, they have shown substantial improvement in performance and significant reduction in system resource requirements.
\end{abstract}

Index Terms-Big Data, Compression, MapReduce, Distributed File System.

\section{INTRODUCTION}

B IG data is now at the frontier of research, innovation and business. The real-world value of big data is evident in a wide range of applications as demonstrated by successful examples including banking [1], healthcare [2], education [3], government [4] and social science [5].

As a valuable asset, data is constantly being collected and accumulated from almost all aspects of life. The term datafication [6] was coined to capture this phenomenon. It is predicted that the global datafication process will generate 44 zetabytes of data by 2020 compared to 4.4 zetabytes in 2013 [7]. It should also be noted that a substantial amount of data being collected is semi-structured or unstructured [8], primarily in text format [9]. The sheer volume of data and the aggressive speed of data growth present major challenges to all aspects of big data analysis from analytic platforms to scalable algorithms. In order to deal with the challenges brought by big data, there is very active ongoing development of new tools, algorithms, computational frameworks, analytic platforms and deployment strategies. Current big data analyssi optimization focuses on scaling up/out analytic platforms [10] [11] and using approximation algorithms [12] [13].

Another optimization, complementary to those other optimizations would be to reduce the size of the source data through compression, and support the direct use of the compressed data in analysis. This aspect of leveraging compression for big data analysis in distributed environments has not been given much attention. On current analytic platforms, such as Hadoop, the purpose of compression is to reduce data size in order to save storage space

- D. Dong is with the Boole Centre for Research in Informatics, University College Cork, Ireland. E-mail: d.dong@cs.ucc.ie

- J. Herbert is with Department of Computer Science, University College Cork, Ireland.E-mail: j.herbert@cs.ucc.ie and lower the data transmission cost over the network. Currently, compressed data cannot be directly involved in MapReduce-based computation. In order to carry out an analysis, the compressed data must firstly be decompressed fully in a sequential manner - sequential due to the fact that modern compression schemes often employ data transformation and/or contextualization techniques which create strong dependencies within the compressed data. The decompression process also requires the underlying storage system to maintain sufficient free space for holding the decompressed data. Additionally, depending on the compression algorithms, decompression speed varies greatly. This can delay considerably the delivery of analysis results when the data volume is large. It should also be noted that previous research has identified that the Input and Output (I/O) system (including both disk $\mathrm{I} / \mathrm{O}$ and network $\mathrm{I} / \mathrm{O}$ ) is often the bottleneck for data-centric analysis in a distributed environment [14] [15]. The network I/O efficiency can be improved by compressing the intermediate data that is going to be transmitted over the network. The disk I/O efficiency can also be improved if the compressed data can be decompressed in parallel in memory as data is being consumed at each computational node of Hadoop (parallel decompression in memory). In this way, the total decompression time can be reduced in proportion to the number of parallel processes. The data loading time can thus be kept to a minimum, and the use of extra storage space can be avoided.

However, if data is to be decompressed in memory, in parallel, in a distributed environment, we will have to ensure that the compressed data is splittable and that each data split is self-contained. This also requires maintaining logical completeness, with respect to the data contents, for each data split. This is because, in general, computational frameworks, such as MapReduce and Spark, process data in 
parallel independently on a per split basis in general; each data split is further broken into a group of logical records (what constitutes a record is data- or application-specific, for example, a record can be a sentence in a plain text file or a row in a database table); a parallel process then deals with a single record at a time. Thus, new compression schemes are needed. This must be done by making the compression process aware of the organization of the data contents, controlling the use of contextual data, and developing appropriate packaging mechanisms so that compressed data is splittable to the Hadoop Distributed File System (HDFS) and MapReduce framework, while also maintaining the logical completeness of each data split to the computational framework without sacrificing too much compressibility.

In the usual MapReduce work-flow, during analysis, a MapReduce program will process data in its original format. This is because MapReduce developers understand the logic and organization of the original data contents to be processed. Also, many existing algorithms and software packages used for parsing the data are designed to work with specific data formats. This also drives exploration of appropriate compression methods for compressing data in such a way that the compressed data can be directly processed in MapReduce without decompression, while ensuring the compressed data is compatible with existing algorithms and software packages and requires minimum effort from MapReduce program developers.

However, processing compressed data poses non-trivial technical challenges, especially in a distributed environment. Traditional solutions to this question are mainly based on indexing techniques [16] [17] [18]. They are developed for traditional information retrieval systems and focus on random access to, and querying of, immutable data content with a cost of high complexity and a constraint on being application or domain specific. Since big data analysis often requires manipulating data, this requires a special data compression where the element of information being compressed is independent of its context, allowing the compressed data to be freely manipulated.

Note that, to improve disk I/O performance, data size needs to be reduced as much as possible. Inevitably, this means employing high compression schemes, such as Gzip and Bzip, which are generally based on transformation and/or contextualization compression techniques. On the other hand, in order to allow a MapReduce program to process compressed data without any decompression, a context-free compression scheme must be used. The fact is that we cannot apply two different conflict compression schemes to a single dataset at the same time. However, observations have indicated that the two approaches can be employed at different stages (data loading and data processing) of MapReduce processing. Thus, designing a layered architecture so that a MapReduce program can, at different stages, take advantage of the corresponding compression layers. In other words, we firstly compress data using a context-free compression scheme and then apply a customized modern compressor to the compressed data. As a result, we can achieve data compression ratios close to the Bzip compressor and gain a substantial improvement on analysis performance over using original data.

Our contributions lie in the design and concrete im- plementation of novel compression schemes for improving performance and reducing resource requirements for big data analysis. In Section 2, we discuss current compression schemes developed for textual big data, followed in Section 3 by a presentation of the proposed compression scheme with detailed explanations. The integration of the proposed scheme and Hadoop is explained in Section 4. The proposed scheme is then evaluated for several standard MapReduce jobs with a collection of real-world datasets using an on-site Hadoop cluster, presented in Section 5. Finally, we summarize our work and indicate future directions in Section 6.

\section{BACKGROUND}

We have witnessed a rapid growth in research and development on big data technologies. Important current concerns include efficient algorithms, parallel computational frameworks, comprehensive analytic platforms, scalable deployment strategies, and auxiliary services that contribute to an effective big data ecosystem. However, related literature on incorporating compression schemes with big data analysis is limited. Current emerging techniques for compressing big data are mostly ad-hoc approaches optimized for data-specific and domain-specific datasets. They are not provided as principled solutions. In this section, we firstly analyze how big data is organized in modern distributed file systems, specifically the Hadoop Distributed File System (HDFS) [19], followed by explaining how big data is processed using the MapReduce computational framework [20]. We then discuss recent literature on emerging techniques including splittable compression, probabilistic data structures and data de-duplication compression.

\subsection{Big Data Organization in Hadoop}

Hadoop is a widely adopted data analysis ecosystem. It consists of several components that together provide a platform for managing and analyzing data on a large scale.

Typically, a Hadoop cluster consists of a group of physical servers in which one of the servers acts as a controller of the cluster, namely the NameNode, and the other servers provide storage space and computation power, namely DataNodes. The NameNode is responsible for coordinating MapReduce jobs and managing HDFS Meta-data. The DataNodes are responsible for storing data and providing computation power for MapReduce programs. Each DataNode contributes a part of its local storage space (e.g., a partition of the local hard disk) to HDFS. Thus, HDFS is a virtualized storage system that is comprised of a number of geographically distributed physical hard disks. A file stored in HDFS is split into a series of fixed-size data blocks. Data blocks and their replications are distributed across DataNodes, but are virtually continuous in HDFS. Unlike the traditional organization of storage systems such as NTFS and Ext3/4 that uses $4 \mathrm{~KB}$ data blocks, HDFS uses a very large data block size (128MB by default) chosen for better hard-disk read/write performance.

\subsection{Data Analysis in MapReduce}

HDFS only provides a logical view of data and hides the complexity of organizing data in the underlying distributed 
storage system. It is an independent service (file system service) provided as a part of Hadoop for data organization and management. Data analysis is carried out by the MapReduce computational framework. As the name implies, a MapReduce program consists of two phases: Map and Reduce. The Map phase operates on a set of key/value pairs. The Reduce phase reduces the outputs from the Map phase by values which share the same key. Each Map process will be assigned to a data block. Moreover, a data block is further broken into logical records (this depends on the dataset, for example a logical record can be a sentence in a text file or a row in a database table). The record parsing process is done by the MapReduce Record Reader component. A Map process will deal with a record at a time. However, HDFS simply splits data by size. It is very likely that a data block will contain incomplete records at the boundaries. To ensure record completeness, MapReduce further organizes data blocks into data splits. Essentially, a data split is a logical view of a data block which is guaranteed to contain a set of complete records.

Intermediate output data from each Mapper is firstly cached in an in-memory buffer. When the utilization of the caching buffer reaches a certain threshold, all cached data in the buffer will be materialized to persistent storage (local hard disks, but not part of HDFS). This process is called Spilling in the context of MapReduce. Data in a Spill will then be sorted by keys and partitioned according to the number of Reduce processes configured for the job. Upon completion of the Map processes, each sorted data partition will be distributed to a corresponding Reducer over the network. This intermediate data distribution process is referred to as Shuffling. At each Reduce node (the physical server that runs the Reduce phase of the program), the received partitions will be sorted again and merged into a single data block, and then fetched to the Reduce process. The final results (Reduce output data) will be stored in HDFS eventually.

\subsection{Data Compression}

Considering the dynamic nature of the compressed contents, block-based compression will work favourably for HDFS, because each compressed block can be made selfcontained. Due to the variable size of the compressed blocks, an additional indexing process must be applied to the compressed contents to log the block sizes, so that the MapReduce Record Reader component (referring to [21]) can effectively and safely parse records. In principle, any block-based compression can be indexed. In practice, LZOsplittable [22] used by Twitter implements such an approach. LZO-splittable uses standard LZO for data compression, then uses a separate program to scan through the $L Z O$ compressed data and log the block boundaries in separate files. In order to consume the LZO-splittable compressed data, a MapReduce program will need both the compressed data and the log file(s). If a dataset contains several files, each compressed file must be associated with a dedicated log file. This makes a MapReduce program more complex and errorprone due to the tracking of block boundaries.

As well as of LZO-splittable, [23] builds inverted file indexing on compression blocks. This allows a MapReduce program to quickly identify desired information in a compressed block without decompression. On the other hand, the LZO compression scheme is speed-oriented. It compresses data at a relatively low ratio. Adding extra indices to the compressed data makes the aggregate compression ratio even lower. More importantly, as Mappers are independent processes, partitioning and distributing indices while maintaining data locality can be very problematic. Hadoop $++[24]$ is other independent work on indexing big data for MapReduce. Hadoop++ does not relax the HDFS storage burden as indices are built on top of source data. Moreover, indices are built when source data is being uploaded to HDFS. This implies that the indices on source data are static. The same indices my not suit different types of analysis jobs. In contrast, [25] provides an adaptive indexing technique for HDFS. Indices are built gradually during the Map processes. The main drawback is that sharing the adaptively built indices across clusters may cause synchronization problems. The majority of those compression methods developed for Hadoop and MapReduce are targeted on source data. They provide better performance for specific types of MapReduce jobs at a cost of more storage space and higher complexity.

In big data query systems, column-wise compression [26] [27] [28] is commonly seen. [26] uses a method that simply compresses column data into a series of self-contained blocks. A group of in-order blocks are then packed into HDFS-Blocks. This makes the compressed data splittable. However, coordinating column-wise blocks horizontally (columns aligned in rows) during data processing can be very difficult. Another scheme, Llama [27] allows groupedcolumn compression with different schemes best suitable for groups of records, and builds indices for compression blocks. Hive [28] is a data management system that allows column-wise compression. Data is organized like database tables in Optimized Record Columnar (ORC) format which is specific to Hive. Internally to ORC, data is partitioned into groups of records, namely stripes. Within each stripe, records are separated into columns. Each column is compressed twice. The first level compression is based on a dictionary method. This is because data in the same column is considered to have similar attributes and therefore shares a common vocabulary. Using a dictionary method can achieve better compression. The first level compression results are then forwarded to the second level compression which uses general purpose compressors such as LZO to pack data into fixed-size blocks. Hive ORC can potentially achieve high compression ratios and it is splittable. A concern is that ORC is data agnostic. Many datasets cannot simply be formatted in columns. For example, XML and JSON formatted data often contains nested records.

Besides indexing techniques, recently, we have seen the use of probabilistic data structures and associated algorithms for dealing with big data in computational biology [29] [30]. Probabilistic data structures are based on Bloom Filters [31] which provide an efficient way of verifying whether a given item exists in a dataset. Using Bloom Filters can significantly reduce data size as a group of messages will be transformed into a single finite series of bits. However, the information transformation is a one-way process. In other words, we can query a Bloom Filter, but we cannot retrieve information that has already been stored in 
it due to the use of hash functions. This limits the applicability of probabilistic data structures to domain-specific use only, such as Genome Sequencing. Another application of probabilistic data structures is big data queries, for instance, BWand [32] for fast query on Twitter tweets, content filtering in MapReduce programs [21], and NoSQL databases such as Google BigTable, Apache HBase and Apache Cassandra. In these cases, the probabilistic data structures are used as an indexing technique to quickly locate information in a distributed storage system. Yet, the original datasets are still needed. There are many other constraints such as immutability and uncertainty that limit the scope of using probabilistic data structures in big data analysis, for example, when modifying the original messages or reading financial records. Generally, probabilistic data structures are suitable for assisting queries on large datasets and applications that can tolerate errors.

Data de-duplication is another popular compression technique used for compressing textual data. Traditionally, it is used in storage systems for backup services and software patching. The basic idea of data de-duplication is to organize information in a hierarchical structure in which the commonality of information flows from top to bottom. It is recently becoming popular for big data due to achieving very high compression ratios. Industry applications such as Google Drive, Dropbox, and RainStor [33] heavily rely on data de-duplication for saving storage space. RainStor has demonstrated that using composite de-duplication schemes can reduce data size by up to $97 \%$. Although, the achievement on compression ratio is surprisingly good, retrieving original text can be time consuming due to the need to go through several de-duplication processes from byte-level to field-level.

In summary, emerging techniques developed for big data mainly focus on reducing data size for saving storage space. In general, these techniques are data- or application-specific schemes which often result in much higher compression ratios as the algorithms are optimized for the particular data with respect to format and contents, compared to general purpose compressors. This makes the current emerging compression schemes uni-functional. By the same token, conventional compression schemes (e.g., Gzip and Bzip) endeavor to achieve higher compression ratios by leveraging preprocessing techniques and/or data contextual information. As this process creates strong dependencies, data accessibility is forced to be sequential. In order to consume compressed data in a seamless fashion, in a distributed environment, an effective compression algorithm must fulfill the requirements of being splittable and allow random access without sacrificing too much compressibility.

It should also be noted that Unicode is the dominant encoding scheme on the Internet at present. The majority of textual contents are encoded in, for example, UTF- 8 or UTF-16 format. Current schemes do not deal with Unicode data effectively. More importantly, these schemes compress data without concern for the organization and format of the underlying data. This makes the compressed data nontransparent to the existing analysis algorithms and software packages. Therefore, new compression schemes, that are content-aware and able to effectively deal with Unicode contents, are needed.

\section{Compression Scheme}

In this work, we introduce the Record-aware Partial Compression (RaPC) scheme suitable for textual big data analysis in Hadoop. RaPC is an embedded two-layer compression scheme. Each layer is designed for the corresponding stage of data processing. The design goal for the outer layer compression scheme is to maximally reduce the data size for minimizing data loading time while making the compressed data splittable to the HDFS. It is based on a modified Deflate algorithm [34]. The inner layer is a word-based, contextfree compression scheme which makes the compressed data directly consumable by MapReduce programs using RaPC supporting libraries.

\subsection{RaPC Layer-1 Compression}

The RaPC Layer-1 (RaPC-L1) encoding is a byte-oriented, word-based partial compression scheme. RaPC-L1 separates informational contents and functional contents. Any character or group of consecutive characters from the range [a $\mathrm{z}],[A-Z]$ and [0 - 9] are considered to be informational contents; other characters are treated as functional contents. RaPC-L1 only compresses informational contents.

The RaPC-L1 code length grows from one byte to the maximum of three bytes depending on the number of compressible strings in the text. A compressible string is a string that is firstly categorized as informational content. Secondly, its length must be longer than the current codelength (because code-length grows dynamically during the compression). Functional characters are used as delimiters to split informational contents. Every unique compressible string will have an integer value assigned to it in the order of their discovery. The integer value is then used to fill in the coding templates. The coding templates consists of one to three bytes with the Most Significant Bit (MSB) of each code-byte set to one. This ensures that the code-bytes are distinguishable from uncompressed contents. For example, given a message "Big Data Analysis", the encoded message is "10000000*00100000*10000001*00100000*10000010". During the compression, the three strings are discovered in order. Their corresponding codes are therefore the integer values 0 , 1,2 . The integer values will be used to fill in the coding template and subsequently replace the corresponding strings in the compressed message. The byte " 00100000 " is the whitespace character defined in the standard ASCII scheme. It is not RaPC-L1 compressed, but used as a delimiter to split strings. The asterisk symbols are only used to indicate byte boundaries for clear presentation in this example. In addition, Unicode characters often use the extended ASCII codes which have the MSB set to one. In order to avoid conflict, Unicode characters are enclosed with a pair of special characters $(0 \times 11$ and $0 \times 12)$. These characters are never used in text data, therefore, it is safe to use them for RaPC-L1 compression.

The RaPC-L1 encoding scheme offers a code space of size $2^{21}$. Before the actual compression process starts, a lightweight sampling process is carried out to gather statistics on the most frequently used words. The top 27 words will then be selected. Each selected word will have an integer value assigned to it. This guarantees that these most frequently used words from a given text have the shortest 
code of one byte. Increasing the number of selected words and/or the sample size will improve the compression ratio. Compression speed will be slower, however, since the process of sorting words by frequency requires $\mathrm{O}(\mathrm{nlog}(\mathrm{n}))$ time, where $n$ is the number of words observed from the samples to be sorted. During the compression, compressible strings and their corresponding codes are temporarily stored in a HashMap data structure. Thus, searching for existing strings is $\mathrm{O}(1)$ complexity. The compressible strings will be replaced by their corresponding code-byte(s) and non-compressible strings will be sent to the output intact. This gives the RaPCL1 compression sub-linear complexity in time.

RaPC-L1 generates two output files: one is the compressed file(s), another is the compression model. The separation of the compressed data and the model are driven by the MapReduce framework. In a MapReduce program, Map processes are independent of each others (the same applying to Reduce processes) in general. For many cases in textual data analysis, the textual contents often need to be converted into other data types (e.g., text to integer values, yes/no to boolean values, and strings to dates). This requires transforming the compressed data into its original format, then converting to other data types accordingly. Because each Mapper loads and processes its assigned data blocks independently, this requires the compression model file to be available to all Mappers and/or Reducers when it is needed. Technically, the HDFS Distributed Cache can be the place for storing the compression model file. The compression model file is a list of compressible strings collected during compression. The index of each string in the list is determined by its corresponding code converted to an integer value. The RaPC-L1 decompression process reads the compression codes and converts them into integer values. Based on the values, we can quickly identify their corresponding original strings. Also consider that data is partially compressed by RaPC-L1, therefore the RaPC-L1 decompression has also sub-linear complexity in time. Another point worth noting is that the compression model file can be reused for compressing future data. If data files are generated from the same source, they often share a common vocabulary, as, for example, data generated by machines.

Recall that the RaPC-L1 scheme compresses data partially. Functional characters such as white-space, comma and new-line are left untouched. This unique feature allows a program to manipulate the compressed data freely, for example, splitting fields by a comma, removing words, and adding new contents etc. Furthermore, it guarantees the manipulated contents are still decodable. However, it should be noted that using the RaPC-L1 compressed data on Hadoop poses a concern. Hadoop currently only supports ISO-88591 and UTF-8 encoding for text. The RaPC-L1 compressed contents are no longer in a traditional text format. In another words, the data cannot be simply converted to strings (e.g., UTF-8 encoded format). This is because of the irregular use of the extended ASCII characters (MSB set to 1). Thus, the RaPC-L1 compressed data must be treated as byte sequences and processed as byte sequences.

\subsection{RaPC Layer-2 Compression}

The RaPC Layer-2 (RaPC-L2) compression can be used with the original text or used to compress the RaPC-L1 compressed data. The purpose of RaPC-L2 is to maximumly reduce data size and package logical records into fixedlength blocks which makes the compressed data splittable to the HDFS.

In the design of RaPC, the aim is to reduce the data size as much as possible. This is driven by the fact that data size is the predominant factor affecting the overall performance of MapReduce programs. Also based on the results of our previous study comparing speed-oriented and space-oriented compression algorithms [14] and our preliminary study RaPC scheme [35], the decompression time is insignificant compared to the time used for loading data from hard disks to memory. RaPC Layer-2 needs to be a scheme that is best the balance between compression ratio and speed. Gzip is the best candidate for RaPC-L2 and it is based on the Deflate algorithm. Thus, the RaPC Layer-2 compression is based on the Deflate algorithm.

The conventional Deflate algorithm is block-based [36]. It consists of a dictionary method (LZ77 [37]) and a statistical method (Huffman Coding [38]). The basic algorithm works as follows. It takes a stream of input and moves the data through a Sliding Window buffer (32KB by default). It starts by checking if there is any string with length up to 258 bytes in the Sliding Window matching the string starting from the current position (the position in the Sliding Window) backwards up to a length of 258 bytes maximum. The longest match wins. The matched string will be replaced by a literal $l$ and a pair of values. The literal is the immediate succeeding character of the matched string. The first value of the pair is the length of the matched string $m$; the second value is the distance $d$ from the current position to the matched string. Then, the Deflater advances $m$ characters in the input stream. If there is no match, the algorithm moves one character forward. This process iterates until the current input buffer is exhausted or the Deflater decides to start a new block of input when the current Huffman trees become inefficient.

The output from LZ77 is a set of literals and pairs of values " $l(m, d)$ ". The Deflate algorithm splits them into two columns. The literals and value $m$ are encoded by a Huffman tree, the distance $d$ is encoded by a separate Huffman tree. Both encoding results are merged and prepended with the two Huffman trees (both of the Huffman trees will be further encoded by the Huffman algorithm) for the final outputs. Each output block corresponds to an input block. In principle, if each output block is self-contained and the boundaries of each block can be recorded, the default Deflate output data could be splittable to the HDFS. But, the Deflate algorithm tends to use the contents of the previous input buffer for the Sliding Window of its immediate succeeding input block in order to improve the compression ratio. These linkages between the input blocks create dependencies between the output blocks and cause the decompression process to be sequential as shown in Figure 1 (upper). If we simply remove these linkages, the overall compression ratio will degrade significantly.

In the RaCP-L2 scheme, we design a higher level buffer with a much larger and fixed size. We refer to this buffer as L2-Block and a Deflate output data block as DO-Block to avoid confusion. The L2-Block is used to accommodate a variable number of DO-Blocks. We force a break in the 


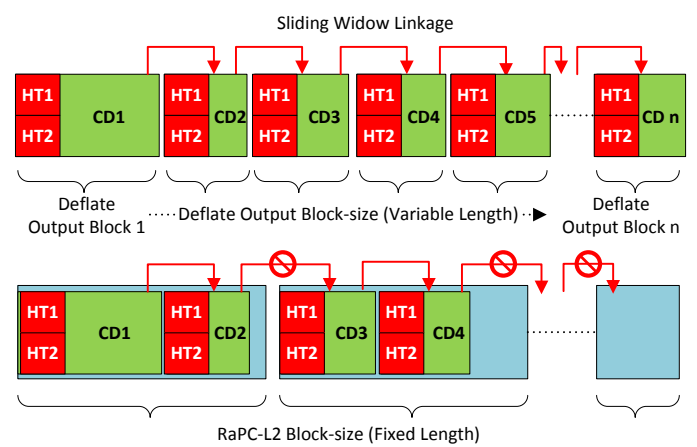

Fig. 1. RaPC Layer-2 block packaging.

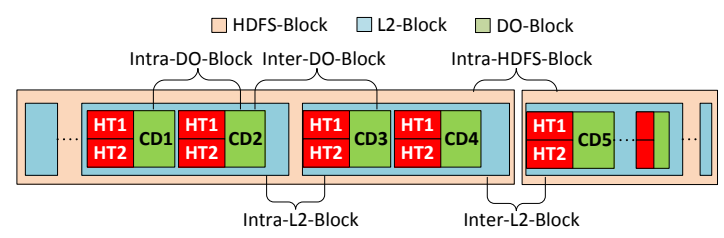

Fig. 2. RaPC Layer-2 data organization hierarchical structure.

linkage between the last DO-Block in the current L2-Block and the first DO-Block in the succeeding L2-Block as illustrated in Figure 1 (lower). Thus, the compressed data in each L2-Block is completely self-contained (each L2-Block can be decompressed independently) which makes the RaPCL2 splittable to the HDFS. The size of the L2-Block strikes a balance between the MapReduce analysis performance and the compression ratio. It will be further discussed in Section 5 .

Moreover, recall that, in the MapReduce framework, the Record Reader supplies one logical record to a Mapper at a time. If we blindly compress data per block, it is very likely that the beginning and/or end of each Deflate input block (DI-Block) will contain incomplete record(s). An example is given in Figure 3 (upper). This makes the Record Reader complicated and inefficient at runtime in either of the two possible scenarios.

Scenario 1: the first scenario (Intra-L2-Blocks) is about determining record completeness between two adjacent DOBlocks in an L2-Block (Figure 2). In Hadoop, each HDFSBlock is processed by a dedicated Map process. The Record Reader that serves the Map process will firstly decompress one L2-Block (containing a number of Compressed Data CD blocks) at a time. Before fetching the decompressed data to the Map process, the Record Reader needs to determine whether the data contains partial records at both the beginning and end of the data. In fact, without decompressing the immediate succeeding L2-Block, we cannot be sure whether the last record is partial or complete. Therefore, we must remove and temporarily store the last record from the current decompressed data, then wait until the next L2Block is due for processing.

Scenario 2: the second scenario (Inter-L2-Blocks) is about

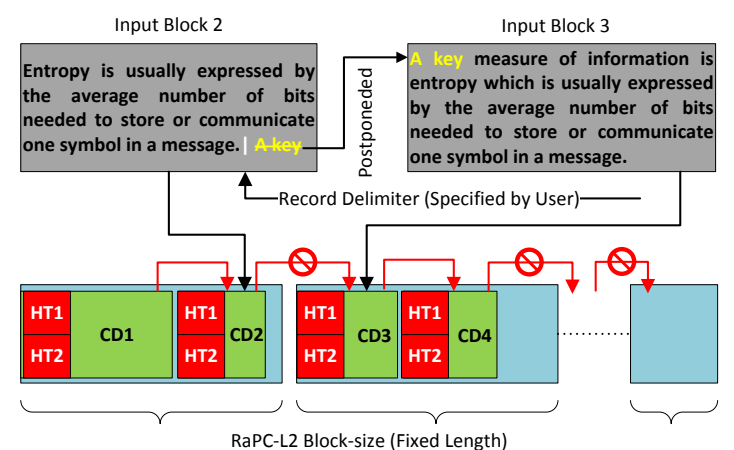

Fig. 3. RaPC Layer-2 record-aware compression.

determining record completeness between L2-Blocks (Figure 2). Recall that Hadoop HDFS organizes big files by splitting them into a series of fixed-size blocks (HDFS-Block). A series of HDFS-Blocks are distributed across the Hadoop cluster Data Nodes. There is no guarantee that logically adjacent HDFS-Blocks will be stored consecutively and/or on the same physical hard disk. Both the L2-Blocks (referring to Figure $2 C D 4$ and $C D 5$ ) belong to separate HDFS-Blocks and are possibly on different physical Data Nodes. The L2-Block (containing CD5) needs to be streamed to the current Map node (which is processing the L2-Block containing CD4) and decompressed. Then, record completeness must be checked. Both scenarios are time consuming and error-prone.

To remove this complication and improve the MapReduce work-flow efficiency, we ensure record completeness at the boundaries of each L2-Block during the data compression phase, hence making it record-aware. What constitutes a record is often dataset specific. To determine records, record delimiters must be given at the beginning of the data compression. At the last DI-Block (corresponding to the last DO-Block) of each L2-Block, we check for record completeness. Any partial record will be postponed to the next DI-Block which will eventually be packed into the next L2-Block. This process only occurs between DO-Blocks. An example is shown in Figure 3. Complex delimiters can be expressed in Regular Expressions. The compression scheme guarantees that each L2-Block contains a set of complete logical records.

As we have noted, DO-Blocks have variable length and L2-Blocks have fixed size. When packaging a number of consecutive DO-blocks in a L2-Block, there is no guarantee that the L2-Block will be filled up exactly. We need to define a packaging format to tell RaPC how much of the payload is contained in the L2-Block. The gaps at the end of each L2Block are filled by trailing bytes. The last two bytes of the L2Block are reserved and used to indicate how many trailing bytes are used. In addition, the MSB of the second last byte is used to indicate whether there is a giant record that spans multiple L2-Blocks. This is designed for an occasion when a dataset contains very long records. Overall, the trailing byte indicator can only allow $2^{15}=32 \mathrm{~KB}$ of trailing bytes to be inserted. In the case where there are only a small number of DO-Blocks left for packaging at the end of the compression, the number of trailing bytes needed may exceed this limit. 


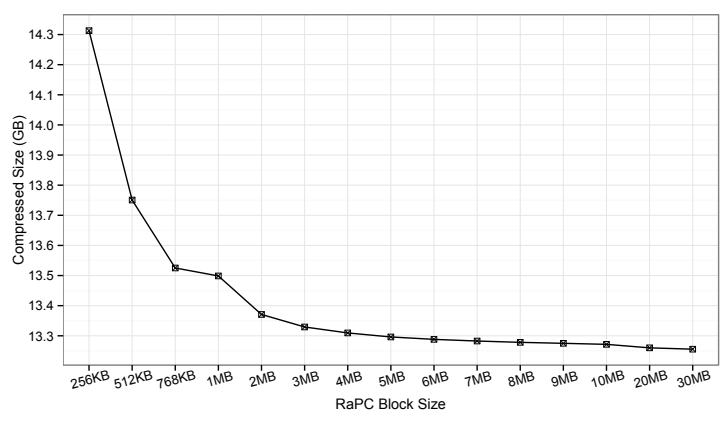

Fig. 4. RaPC Layer-2 block size verses compression ratios

For this reason, an exception is made so that the size of the last L2-Block is the total length of the last several DO-Blocks exactly. That is, no trailing bytes are inserted into the very last L2-Block.

Note that an increase in L2-Block size will slightly improve the compression ratio. Figure 4 shows the results from compressing the dataset DS-WikiEN (the original dataset is $47 \mathrm{~GB}$, referring to Table 4 ) with various L2-Block sizes. In this experiment, we start with a L2-Block size of $256 \mathrm{~KB}$ and gradually increase the block size to $30 \mathrm{MB}$. In Figure 4, we observe a rapid drop in compressed data size (increased compression ratio) from $256 \mathrm{~KB}$ to $2 \mathrm{MB}$, and show decrease in compressed data size when the L2-Block size is beyond $2 \mathrm{MB}$. This is due to two reasons.

Firstly, we break the Inter-DO-Block linkages to make the compressed data splittable. This implies that the current DI-Block cannot use the information from its immediate predecessor for the contents of the current Sliding Window. The disconnection leads to the compression for the current DI-Block to accumulate its own new context, and at the beginning of this context accumulation phase, much less information can be compressed. This results in a lower compression ratio.

In addition, the Sliding Window is $32 \mathrm{~KB}$ by default, so that in the worst case scenario, if no information can be compressed during the context accumulation phase, there will be $32 \mathrm{~KB}$ of incompressible data at the beginning of every L2-Block. When the L2-Block size is small, the $32 \mathrm{~KB}$ incompressible data will occupy a relatively large proportion of the total $\left(\frac{32 K B}{L 2-B l o c k s i z e}\right)$ and thus have a bigger influence on the compression ratio. This is the main cause of the rapid drop in the compressed data size (increase in compression ratio) as the L2-Block size increases.

Secondly, during the L2-Block packaging processes, trailing bytes and indicator bytes must be appended to the last DO-Block to fulfil the requirements for a record-aware L2Block. By increasing the L2-Block size, we have statistically reduced the number of trailing bytes and indicator bytes needed, thus improving compression ratio.

This experiment gives us a general idea how the L2Block size affects the compression ratio. The results in Figure 4 may vary slightly depending on the contents of a given dataset. Choosing a bigger L2-Block size can improve compression ratio, but it has side-effects on MapReduce program performance. This will be studied further in Section 5 .

\section{RaPC ON Hadoop}

In order to use RaPC compressed data on Hadoop with maximum transparency to MapReduce programs and developers, we provide a set of utility functions including: a customized RaPC Record Reader for decoding the RaPCL2 compressed data; a RaPC TextWritable data type which implements equivalent operations found in the Hadoop Text data type for handling the RaPC-L1 compressed data; and a SequenceFileAsBinaryOutputFormat Record Writer for writing RaPC-L1 compressed data and general binary data to the HDFS.

The RaPC work-flow is as follows. The RaPC compressed files must be stored in the HDFS, and if it is needed, the RaPC-L1 compression model file must be loaded to the HDFS Distributed Cache. A MapReduce program must set the provided record reader (RaPCInputFormat) as the default input format class. This ensures that the RaPC Layer- 2 compressed data can be correctly read and decoded. The input value type for Mappers must be set to RaPCTextWritable or the regular Text type. If the data is compressed by RaPCL2(L1) (RaPC-L1 embedded in RaPC-L2), then the records received by the Mapper are in fact a block of binary data in the RaPC Layer-1 compressed format, and the RaPCTex$t$ Writable data type must be used. If the data is compressed by RaPC Layer-2 only, the decoded data is the original text, and then the regular Text data type (in the context of the MapReduce framework) can be used.

For many data analysis jobs, parsing data records is necessary. For the original text, manipulating data is simple as the data contents are readable by developers. In RaPC, we provide a transformation function $T()$ that converts between original text and RaPC-L1 compressed data. For example, if we write a regular Java clause for searching a phrase "value.contains("big data");", then in RaPC it is simply "value.contains(RaPC.T("big data"));". In this example, the variable "value" in the regular program can be a String data type. In RaPC, the variable "value" needs to be declared as a RaPCTextWritable data type. The "contains( )" function in RaPC is an implementation of the "contains( )" function found in the regular Java String class for the RaPCTex$t$ Writable data type. Indeed, the transformation requires the RaPC-L1 compression model file to be loaded at the Map and/or Reduce initialization phase. This may incur a small delay due to loading the model files from HDFS Distributed Cache to the local computational nodes. For some types of job, for example, $N$-Grams analysis, there is no need to load model files, and those MapReduce programs can take full advantage of using RaPC.

Another point worth mentioning is that the final MapReduce output can be in the original text format or RaPCL1 compressed format. In the former case, the RaPC-L1 decompression needs to be carried out at each Reducer before writing any results to HDFS. The second case needs more attention. By default, writing binary records to HDFS requires the SequenceFileAsBinaryOutputFormat provided by the MapReduce framework. This default Record Writer also writes some auxiliary information about each binary record including record key/value length and it periodically inserts synchronization points $(0 x F F)$. The record key/value length are directly converted from their numerical values to the 
byte sequences; this makes the final output specific to Hadoop. Furthermore, the synchronization point is a character drawn from the extended ASCII codes which is very likely to clash with RaPC-L1 codes and confuse the RaPC-L1 decompression process. An additional requirement for writing the RaPC-L1 compressed output to HDFS is therefore to use our customized RaPCSequenceFileAsBinaryOutputFormat which has synchronization points removed and has well formatted key/value length.

In general, for a RaPC-L2-enabled MapReduce program, configuring file input format is the only major difference; for an RaPC-L2(L1)-enabled program, we need to load the model file, configure the file input format, output format, and corresponding data types.

\section{Evaluation}

We evaluate the effectiveness of RaPC in an on-site Hadoop cluster. The cluster consists of six nodes. Each node is configured with dual core Intel E8400 (3.0GHz) CPU, 8GB RAM and 1TB SATA3 hard drive. Nodes are connected to a NetGear GS716T Gigabit Ethernet switch. Specifically, the cluster is configured with Hadoop version 2.6.0 and Linux kernel 2.6.32. The HDFS is configured with single replication and 64MB block size. The MapReduce jobs used in the evaluation are summarized in Table 1 . We use several real-world datasets as listed in Table 4 for the evaluation. The evaluations of the RaPC compressor and RaPC with MapReduce include measuring analysis performance, cluster storage requirements, memory constraints and compression performance. In the experiments, the number of Mappers and Reducers are optimized for each job. RaPCL2 block-size was set to $1 \mathrm{MB}$ across all the experiments. We compare RaPC with the state-of-the-art Hadoop-LZO (also know as LZO-splittable), version 0.4.20.

\subsection{Performance}

Table 1 contains our main evaluation results. We use ten different MapReduce jobs to evaluate the effectiveness of RaPC on Hadoop with a range of real-world datasets having different properties. We run each job three times with original, RaPC-L2, and RaPC-L2(L1) compressed data. We record the input data size, intermediate output (Map output) size, final output (Reduce output) size, memory allocation, and analysis duration for each job with the three different input data types.

The Site Rank job is selected to demonstrate the full advantages of using RaPC with MapReduce. In this job, we calculate the rank for each website in the given dataset based on a ranking algorithm defined for an undirected graph. According to [39], given an undirected graph $G(V, E)$, calculating the rank for vertex $v_{i}$ in $G$ is equivalent to calculating the degree distribution of $v_{i}$ defined by $\frac{d\left(v_{i}\right)}{2|E|}$, where $d\left(v_{i}\right)$ is the degree of vertex $v_{i}$ in $G$. Thus the site rank job is to obtain a vector as given by $\frac{1}{2|E|} \cdot\left[d\left(v_{0}, d\left(v_{1}\right), \cdots, d\left(v_{n}\right)\right]\right.$, where the edges $E$ are the connections between websites $V$.

In fact, the calculation part of the Site Rank job is relatively lightweight. The heavy part is parsing the dataset and finding out all of the connections between websites. The MapReduce job involves parsing the URLs for each website, then finding and formatting edges $E$. Using the RaPCL2 compressed data for the Site Rank job, we accelerated the calculation speed by $59.8 \%$ compared to that same job with the original text data. The performance gains come from two sources. Firstly, the RaPC-L2 compressed data is approximately four times smaller than the original data (RaPC-L2 reduces the original data size by $74.3 \%$ ). Loading the RaPC-L2 compressed data from hard disks to memory requires much less time. Secondly, due to the MapReduce Local Combiner effects, the intermediate data is smaller. This reduces the time required to transmit data from Mappers to the Reducers over the network.

Additional to the job with RaPC-L2 compressed data, using RaPC-L2(L1) compressed data can further improve the analysis performance. When parsing the URLs, we need to split each URL using the forward slash character "/". The results of splitting gives an array of sub-strings drawn from the Web link. We are interested in the website link part only. The access protocol and the page identifier parts must be omitted. The string splitting process is about searching for the delimiter "/" and taking the sub-string from the link iteratively. By default, the Java implementation of the string split function invokes the indexOf( ) function to locate patterns which takes $O(m(n-m))$ time, where $m$ and $n$ are the pattern length and source string length, respectively. In this case, $m=1$, therefore each search on pattern "/" takes $O(n-1)$ time. Recall that the RaPC-L1 compression does not compress functional contents. We can search for the forward slash character from the RaPC-L1 compressed data directly without using the model files. The compression ratio for this particular dataset can be found in Figure 6. It is approximately $49 \%$. Theoretically, this implies searching the same pattern from the new source $O\left(n^{\prime}-1\right)$ is approximately twice as fast as $O(n-1)$, where $n^{\prime}=0.49 n$.

In the second job, we use a $N$-gram task for evaluating the $\mathrm{RaPC}$ under intensive disk I/O operations. $\mathrm{N}$-gram analysis is a common technique for speech recognition. We use 5Gram to produce sufficient in-memory data to increase the frequency of memory to disk I/O operations. The job consists of Map tasks only. There are no Shuffling and Reducing phases, thus the network I/O is kept to a minimum. Comparing the results of the original and RaPC-L2 compressed data, RaPC-L2 improves the processing speed by $33.4 \%$. Because both analyses generate the same size output, therefore the main performance gain is from distributing MapReduce data-splits to Mappers. Using RaPC-L2(L1) compressed data can further improve the analysis speed by $\sim 2.2 \%$ because of the smaller input and output data in RaPC-L1 compressed format.

Besides the efficiency, there is a useful side-effect when using RaPC-L2(L1) compressed data. Recall that the 5-Gram job reads, manipulates, and writes data in the RaPC-L1 compressed format. During the lifetime of the job, the RaPCL1 model files are not required. As the RaPC-L1 compression encrypts (encodes) the informational contents, using the RaPC-L2(L1) compressed data can provide a certain level of protection on data privacy in a shared cluster or in a public cloud environment.

The Word Count job is used as a standard benchmark for results comparison. Also, the Word Count job generates comparatively larger intermediate and final output data. Using 
TABLE 1

A summary of MapReduce jobs used for evaluating RaPC on Hadoop.

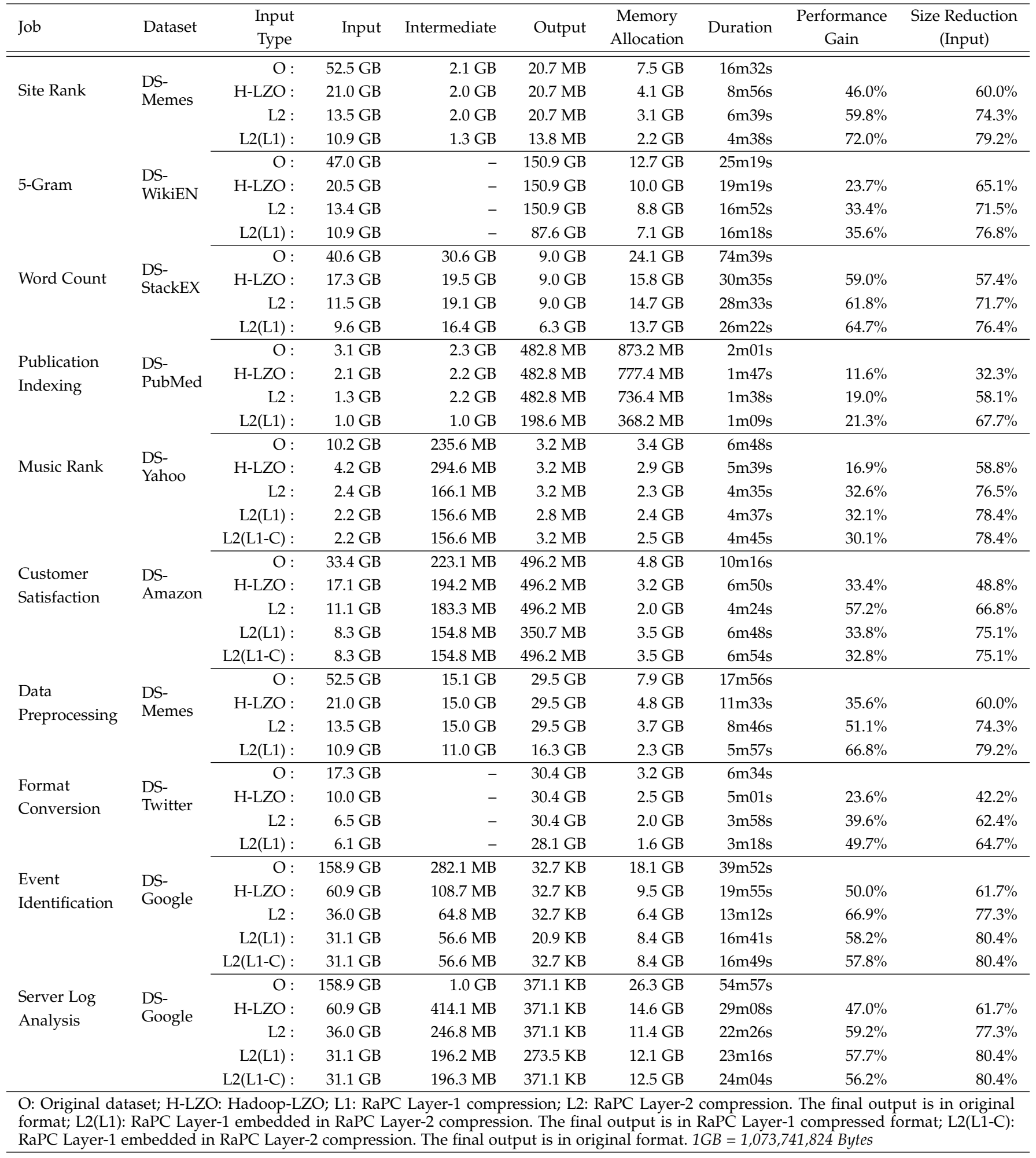

RaPC-L2 or RaPC-L2(L1) compressed data, we can reduce the size of the intermediate output by $33.5 \%$ and $43.5 \%$, respectively. Thus, the transport cost of distributing the Map output to corresponding Reducers over the network (MapReduce Shuffling phase) can be reduced significantly. It also reduces the cost of materializing in-memory data to local persistent storage (local hard disks). The reduction of the size of the intermediate output is due to the MapReduce Local Combiner effects.

Recall the work-flows in the Map phase, and note that the following parameters are the default values in Hadoop version 2.5.0 or above. When processing a data split, the 
Map outputs are temporarily stored in an in-memory buffer (100MB). When the buffer fills to a certain threshold $(80 \%)$, a background process starts materializing the in-memory data to the local persistent storage. During this spilling phase, the buffered data is firstly partitioned according to the number of Reducers configured for this particular job. Within each partition, records are sorted by keys. The sorting results are then fetched to the Local Combiner process to combine the records with duplicate keys. The output of the Local Combiner is called a "Spill" and it is then written to local hard disks. Generally, the data processed by a Local Combiner is much smaller depending on the number of records with duplicate keys found. Thus, we can reduce the time used to write Spill data to disks.

For each individual Mapper, if the generated intermediate data is larger than the in-memory buffer size, each Mapper will produce a series of Spills. Upon the completion of processing a data split, all partitions from the group of Spills that belong to the same Mapper will be merged based on their partition number and then sorted by record keys. The sorting results are again fetched to the Local Combiner to further examine records with duplicate keys. This is the place where the RaPC can reduce the intermediate data size. The reasons are as follows. In any given cluster environment, the HDFS-Block size is fixed. Assume processing each data split will generate $m$ records on average. With the original text data, the Local Combiner process is about finding records with duplicate keys in $m$. With RaPC compressed data, each Mapper will receive a block of data with the same size, but in compressed format. Given the compression ratio $\varphi$, defined as $\varphi=\frac{S_{o}-S_{c}}{S_{o}}$, where $S_{o}$ denotes the size of the source file, and $S_{c}$ denotes the size of the compressed file. Each Mapper will actually receive $\frac{S_{c}}{1-\varphi}$ size data. For instance, in this Word Count job, assuming that the number of Map output records are proportional to the Map input data size, using the original text data, each Mapper will receive 64MB (equal to the HDFS-Block size) data split on average and produce $m$ records. For the RaPC-L2 compressed data with a compression ratio of $71.7 \%$, each Mapper will receive $\frac{64 M B}{(1-0.717)} \approx 226 \mathrm{MB}$ data (after RaPC-L2 decompression) and produce $\sim 3.5 m$ number of records. When $m$ increases, we will have a statistically greater chance of finding more records with duplicate keys and/or more duplicate keys for a record, thus further reducing the size of the intermediate outputs. In general the bigger the size of L2-Block, the more the effects of Local Combiner with RaPC are further enhanced.

In the Publication Indexing job, we calculate the descriptive statistics for each publication based on the importance of the author(s) which is further defined by the number of publications from the author. Thereafter, the index of the publication can be sorted by designated statistics. We use the PubMed database records [40] for this job. The dataset contains $\sim 22$ million publications and $\sim 11$ million authors. Referring to Table 1, RaPC-L2 compresses the data by $58.1 \%$ which is the lowest ratio among the ten jobs. Considering the similar intermediate and final output data size, this low compression ratio leads directly to the low performance gain of $19 \%$. Further improvement of $2.3 \%$ is given by using the RaPC-L2(L1) compressed data which is the combined contribution from the smaller intermediate and final output data size.

TABLE 2

Comparison of Map input records for the original, RaPC-L2 compressed and RaPC-L2(L1) compressed data.

\begin{tabular}{l|r|r|r}
\hline \multirow{2}{*}{ Job } & \multicolumn{3}{|c}{ Number of Map Input Records } \\
\cline { 2 - 4 } & Original & RaPC-L2 & RaPC-L2(L1) \\
\hline \hline Site Rank & 919061195 & 13810 & 11160 \\
\hline 5-Gram & 805750261 & 13823 & 11027 \\
\hline Word Count & 6126845962 & 11745 & 9881 \\
\hline Publication Indexing & 21788173 & 1370 & 1020 \\
\hline Music Rank & 699640226 & 2435 & 2268 \\
\hline Customer Satisfaction & 381554470 & 11397 & 8490 \\
\hline Data Preprocessing & 919061195 & 13810 & 11160 \\
\hline Format Conversion & 468854886 & 6622 & 6232 \\
\hline Event Identification & 1232799308 & 36915 & 31840 \\
\hline Server Log Analysis & 1232799308 & 36915 & 31840 \\
\hline
\end{tabular}

In the Music Rank job, we use the Yahoo! Music Rating dataset [41] which contains a large number of very small records (approximately 16 characters per record on average). The dataset contains $\sim 700$ million ratings on $\sim 136$ thousand songs provided by Yahoo! Music services. The task is to calculate the mean scores and standard deviation for each song. For this task, the MapReduce Record Readers are heavily loaded supplying records to the corresponding Mappers. The default Record Reader provided by the MapReduce framework treats a line as a record. The records processed by a Mapper will have on average 16 characters. This makes the default Record Reader inefficient and wastes a lot of cluster resources such as Java Heap Space (memory) assigned to the Mapper. With compressed contents, our RaPCRecordReader is in fact supplying a set of records to a Mapper at a time. Table 2 shows the number of records received by Mappers for each job with different input data. It works by reading a fixed-size block of data (L2-Block, the size of L2Block is adjustable). The block of data will be decompressed in-memory and then forwarded to a Mapper. Because the L2-Block size is fixed, and more importantly, each L2-Block contains a set of complete records, there is no need to track record length and worry about partial records at the boundaries of L2-Blocks and HDFS-Blocks. This makes the RaPCRecordReader more efficient and lightweight than the default MapReduce Record Reader. Additional to the RaPCL2(L1) compressed data, in order to calculate music scores, we must convert the text contents to real numerical values. This conversion requires loading the RaPC-L1 compression model files to each Mapper. The loading process and the text to numerical value conversion take extra time and occupy more memory. This leads to the job with the RaPC-L2(L1) compressed data being slightly slower than the job with RaPC-L2 compressed data.

The Customer Satisfaction job is a special case. When processing RaPC-L2 compressed data, the job results in a relatively high performance gain of $57.2 \%$. Two sources contribute most to this result. The first source is the lower data transmission cost due to the smaller data size. The second source is the Group Record Effect. Records in this particular dataset are split by empty line(s). Each record consists of multiple fields delimited by a new line character. 
Records contain a variable number of fields. By default, the MapReduce framework treats the new line character as the delimiter. The default Record Reader supplies a line of text to a Mapper at a time. This requires Mappers to wait for the Record Reader until a full logical record is received. The waiting time is the main cause of the delay. With RaPC compressed data, a group of complete records are given to one Mapper at a time, and therefore, it saves on waiting time. Indeed, writing a customized Record Reader using a traditional approach for this particular dataset can improve the analysis performance on the original dataset. But, it will incur extra programming and will still suffer from the big data size.

The Data Preprocessing job is used to evaluate the effectiveness of RaPC-L2(L1) when dealing with highly skewed giant records. In this job, we create records that have a source website link as the key and all web pages that refer to the source website as the value. The preprocessed data thereafter can be used for other calculations, for example, web site popularity and centrality analysis. We can use the Memetracker memes dataset [42]. It contains $\sim 418$ million web page URLs. Some extremely popular websites are referenced by many web pages. Formatting and appending the referring web page links to a source website can result in a giant record. If the size of the giant record is larger than the size of the Java Heap Space that is currently available to the Mapper, it will cause very frequent in-memory data to disk swapping processes. The disk I/O bursts were observed in both the tasks with original text and RaPC-L2 compressed text. This irregular event also causes extra delay to the job completion time.

In contrast, when processing RaPC-L2(L1) compressed data, it is hard to identify any obvious disk $\mathrm{I} / \mathrm{O}$ bursts. Referring to Figure 6, the RaPC-L1 compression ratio for this particular dataset is $\sim 49 \%$. Generally, the Map output records are half the length of those produced by the same tasks with either original or RaPC-L2 compressed text. Additionally, in the previous version of Hadoop, when the record size is larger than the size of the Java Heap Space available to the Mapper, the MapReduce framework will throw Java Heap Space Errors, and the corresponding parameter "mapred.reduce.child.java.opts" needs to be re-adjusted to a bigger value and then the entire cluster needs to be restarted to pick up the changes. The Format Conversion job is similar to the previous task.

The Server Log Analysis and the Event Identification jobs are used to evaluate RaPC with comparatively large jobs. The main task of the Server Log Analysis is to determine the over/under utilized servers from Google Cluster Log files [43]. The dataset contains $\sim 1.2$ billion records in Comma Separated Value (CSV) format. For this particular dataset, RaPC-L2 and RaPC-L2(L1) can reduce the original data size by $77.3 \%$ and $80.4 \%$, respectively. The size of the intermediate output is significantly smaller because of the MapReduce Local Combiner effects discussed above and the highly repetitive nature of the data. Due to the significant data size reduction (both input and intermediate output), we have achieved $59.2 \%$ and $57.7 \%$ performance gains from the job with RaPC-L2 and RaPC-L2(L1) compressed data, respectively. During the analysis, numerical values in the RaPC-L1 format need to be decoded to standard strings

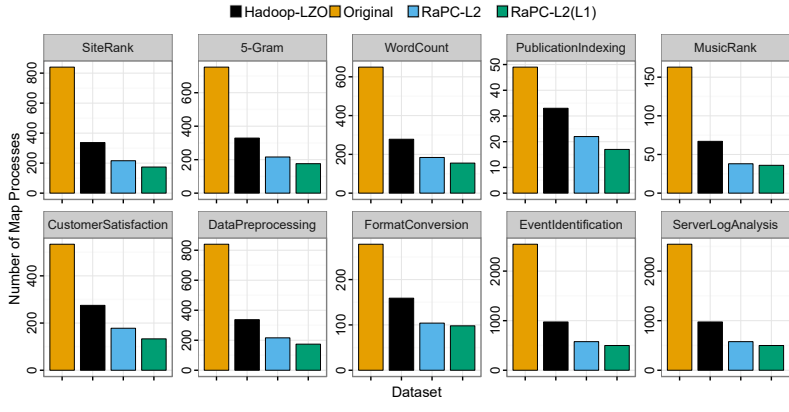

Fig. 5. Number of Map processes for each job (default to Hadoop) with original, RaPC-L2 compressed and RaPC-L2(L1) compressed data.

and then converted into real numerical values. This requires the RaPC-L1 compression model file to be available for each Mapper, which makes RaPC-L2(L1) job slower than the job with the RaPC-L2 compressed data. The level of the performance degradation is influenced by the number of Mappers and how much RaPC-L1 compressed data needs to be decoded during the analysis. If we increase the HDFSBlock size (equivalent to reduce the number of Mappers), we can improve analysis speed and memory consumption, accordingly. Note that the RaPC-L1 compression model file needs to be loaded to each Mapper separately. The smaller number of Mappers results in less memory (aggregated) being used to hold the RaPC-L1 models. Rationally, if a considerable portion of the RaPC-L1 data needs to be decoded during the job execution, then just using the RaPC-L2 compression for the data is a more appropriate approach. This also applies to the Event Identification job.

In general, using the RaPC-L2 or RaPC-L2(L1) compressed data requires much less memory. The reasons are as follows. Firstly, Mappers are independent processes and require dedicated Java Virtual Machines (JVMs). Each has a default and dedicated Java Heap Space assigned to it. The more Mappers the more memory is required. Because the input data size is the dominant factor affecting the number of Mappers, the compressed data size is significantly smaller resulting in a smaller number of Mappers and consequently less aggregate memory consumption. Figure 5 shows the default number of Mappers configured for each job with the original, RaPC-L2, and RaPC-L2(L1) compressed data. Moreover, supplying a block of data to a Mapper at a time can make more efficient use of the memory .

Furthermore, we compare the RaPC scheme with the state-of-the-art Hadoop-LZO. Using Hadoop-LZO compressed data in Hadoop is similar to our RaPC scheme. HadoopLZO compresses data using the standard LZO compressor. The compressed data is then uploaded to HDFS. A special indexer program is used to index and record the splittable boundaries of the compressed data. The output of the indexer is a set of indexing files which are associated with each individual dataset. In MapReduce, each Mapper uses the indexing files to calculate splittable boundaries and take the data block for processing. As the number of datasets increases, maintaining and processing the indexing files can be complicated. The main comparison results are shown in Table 1. In general, using RaPC compressed data 


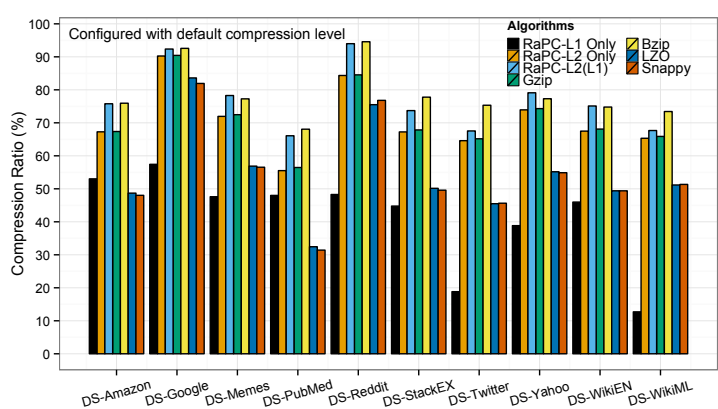

Fig. 6. RaPC compression ratio comparing to Bzip, Gzip and $L Z O$ with default compression level .

can improve MapReduce performance by $13.5 \%$ (RaPC-L2) and $15.1 \%$ (RaPC-L2(L1)), and can further reduce data size by $16.2 \%$ (RaPC-L2) and $21.0 \%$ (RaPC-L2(L1)), on average, compared to that using Hadoop-LZO compressed data.

In brief, we have demonstrated the flexibility and effectiveness of using RaPC with MapReduce using a variety of standard MapReduce jobs. Using RaPC-L2 does not exhibit any shortcomings and RaPC-L2(L1) can further improve efficiency of analysis speed, cluster memory usage, and storage usage. It can be most beneficial to clusters that are I/O bound and for whom storage space is a concern. For some jobs that need many conversions, for example, financial report analysis, using the $T$ ( ) function frequently can affect the overall performance. In those cases, using RaPC-L2 compression alone may be more appropriate.

\subsection{RaPC Characteristics}

In this section, we evaluate the characteristics of RaPC-L1, L2, and -L2(L1) schemes in terms of compression ratio, compression speed and decompression speed. The results are thereafter compared with four common compressors that are currently supported by the Hadoop system, including Gzip (v1.6), Bzip (v1.0.6), LZO (v1.03) and Snappy (v1.1.2). Exactly 2GB of data is drawn from each dataset (as shown in Table 4) for the experiments. There are five runs for each experiment. The compression ratio for Gzip, Bzip, LZO and Snappy are identical across the five runs. In contrast, there are some slight variations in the RaPC-L1, -L2, and -L2(L1) compression results due to the random sampling effects. The compression and decompression speed also varies slightly due to system variation of the testbed. The mean values and standard mean errors are calculated and included in the experimental results. All experiments are carried out on Linux kernel version 3.19 .0 and $\times 86 \_64$ architecture platform with dual WD5000AAKS-75V0A0 500GB hard disks (7200RPMs) and Ext4 (version 1.0) file system.

\subsubsection{Compression Ratio}

Compression ratio is content dependent. Figure 6 shows the compression results for RaPC, Gzip, Bzip, LZO and Snappy. Specifically, Gzip, Bzip and LZO are configured with the default compression level settings. RaPC-L1 can compress data by $\sim 50 \%$ on average. There are two exceptions: dataset DS-Twitter and dataset DS-WikiML. Recall that RaPC-L1 does not compress Unicode contents. Dataset DS-WikiML is multi-language Wikipedia articles, where the majority of the contents are complex Unicode texts which are incompressible. Additionally, we must use the $0 \times 11$ and $0 \times 12$ character pair to enclose any Unicode strings. This leads to the low RaPC-L1 compression ratio on dataset DS-WikiML. Dataset DS-Twitter contains $\sim 490$ million Twitter tweets collected world wide. A large number of records contain Unicode texts. Moreover, each tweet record is comprised of a timestamp, anonymized user ID which is often a hash code, and tweet topics. This makes the vocabulary size for the dataset much larger. Considering that the RaPC-L1 code length increases with the vocabulary size, this makes the average code-length longer for the dataset. In practice, if the the data is known to contain many Unicode strings, it is better to avoid using RaPC-L1 compression at all.

RaPC-L2 is a block-based compression based on the Deflate algorithm. In principle, RaPC-L2 can achieve the same compression ratio as Gzip. Recall that, in RaPC-L2 compression, we intentionally break the connections between DOBlocks and stuff trailing bytes at the end of each L2-Block; this makes RaPC-L2 compression slightly worse than Gzip. In fact, RaPC-L2 compression ratio is very close to Gzip.

RaPC-L2(L1) is a composite compressor. It applies the RaPC-L2 compression on the RaPC-L1 compressed data. In practice, it can achieve compression ratios close to Bzip and sometimes even better than Bzip. For example, the compression results from Bzip and RaPC-L2(L1) for dataset $\{D S-G o o g l e, D S-M e m e s, D S-T w i t t e r, D S-W i k i E N\}$ are given by $\{\{152.2 \mathrm{MB}, 150.5 \mathrm{MB}\},\{465.6 \mathrm{MB}, 442.4 \mathrm{MB}\},\{663.5 \mathrm{MB}$, $663.3 \mathrm{MB}\},\{456.3 \mathrm{MB}, 427.1 \mathrm{MB}\}\}$, respectively.

\subsubsection{Compression Speed}

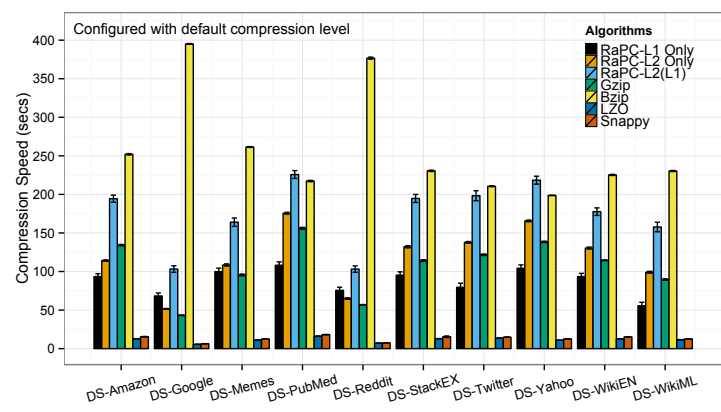

Fig. 7. RaPC compression speed comparing to Bzip, Gzip and $L Z O$ with default compression level .

For compression speed, the current implementation of RaPC-L2 achieves similar speed to Gzip, and RaPC-L2(L1) is similar to Bzip. Figure 6 shows the compression speed with default compression levels configured for Gzip, Bzip and LZO. In general, Bzip is the slowest compressor, because it has an extra step for block sorting. The speed is often influenced by block size. LZO is the fastest on decompression. In contrast, RaPC-L2 and Gzip are relatively stable and consistent. Figure 6 shows the decompression speed for each compressor configured with default compression levels. Furthermore, we compare the RaPC scheme with the state-of-the-art Hadoop-LZO. Using Hadoop-LZO compressed data in Hadoop is similar to our RaPC scheme. HadoopLZO compresses data using the standard LZO compressor. 


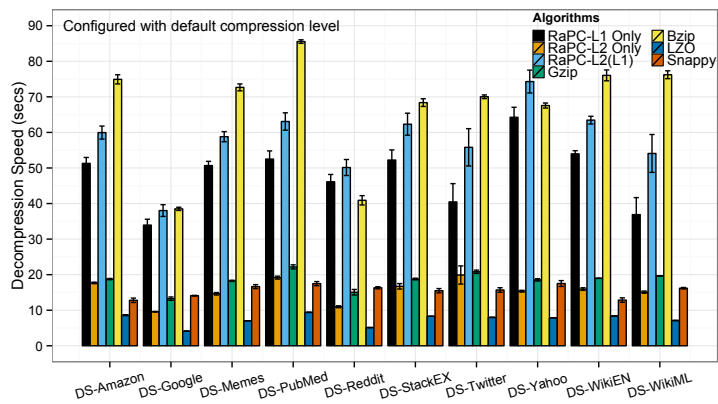

Fig. 8. RaPC decompression speed comparing to Bzip, Gzip and LZO with default compression level .

TABLE 3

Performance of the Hadoop-LZO compression.

\begin{tabular}{l|r|r|r}
\hline Dataset & Compression Time & Indexing Time & Index Size \\
\hline DS-Amazon & $6 \mathrm{~m} 44 \mathrm{~s}$ & $6 \mathrm{~m} 46 \mathrm{~s}$ & $1.0 \mathrm{MB}$ \\
\hline DS-Google & $30 \mathrm{~m} 17 \mathrm{~s}$ & $10 \mathrm{~m} 04 \mathrm{~s}$ & $5.1 \mathrm{MB}$ \\
\hline DS-Memes & $10 \mathrm{~m} 17 \mathrm{~s}$ & $3 \mathrm{~m} 53 \mathrm{~s}$ & $1.6 \mathrm{MB}$ \\
\hline DS-PubMed & $0 \mathrm{~m} 38 \mathrm{~s}$ & $0 \mathrm{~m} 54 \mathrm{~s}$ & $98 \mathrm{~KB}$ \\
\hline DS-StackEX & $8 \mathrm{~m} 19 \mathrm{~s}$ & $2 \mathrm{~m} 53 \mathrm{~s}$ & $1.3 \mathrm{MB}$ \\
\hline DS-Twitter & $3 \mathrm{~m} 34 \mathrm{~s}$ & $4 \mathrm{~m} 10 \mathrm{~s}$ & $555 \mathrm{~KB}$ \\
\hline DS-WikiEN & $8 \mathrm{~m} 38 \mathrm{~s}$ & $4 \mathrm{~m} 41 \mathrm{~s}$ & $1.5 \mathrm{MB}$ \\
\hline DS-Yahoo & $1 \mathrm{~m} 50 \mathrm{~s}$ & $0 \mathrm{~m} 45 \mathrm{~s}$ & $325 \mathrm{~KB}$ \\
\hline
\end{tabular}

A special indexer program is used to index and record the splittable boundaries of the compressed data. The output of the indexer is a set of indexing files which are associated with each individual file in the given dataset. Table 3 shows the performance of the Hadoop-LZO, in terms of compression speed and the size of the indexing file(s). As the number of files increases, maintaining and processing the indexing files can be complicated.

\section{CONCLUSION}

The real value of big data has been gradually realized and boosted recently by successes in both public and private sectors. This encourages decision makers to accumulate more data and perform analysis at an increasingly larger scale. As the ever-increasing volume of data is continuously challenging data analysis tools, algorithms and platforms, data compression is an effective way of reducing data size. However, existing compression schemes do not offer significant benefits to big data analysis. In order to get the maximum value out of using data compression for big data analysis, designing new compression schemes that take into consideration data content, computational model and analytics platform is necessary. In response, we have developed the RaPC scheme which leverages novel compression for improving performance and reducing resource requirements for textual big data analysis in Hadoop. It has been implemented as a full solution supporting ease of use by developers and orthogonal to other possible optimizations. The advantages of the RaPC scheme for textual data analysis have been demonstrated using a variety of standard realworld benchmarks.

\section{References}

[1] T. H. Davenport and J. Dyché, "Big data in big companies," International Institute for Analytics, Thomas H. Davenport and SAS Institute Inc., Report, May 2013.

[2] D. K. Peter Groves, Basel Kayyali and S. V. Kulken, "The big data revolution in healthcare: Accelerating value and innovation," Center for US Health System Reform Bussiness Technology Office, Report, Jan 2013.

[3] V. Kellen, A. Recktenwald, and S. Burr, "Applying big data in higher education: A case study," Cutter Consortium, Data Insight \& Social BI Executive Report 8, December 2013.

[4] V. Morabito, "Big data and analytics for government innovation," in Big Data and Analytics. Springer Science \& Business Media, 2015, ch. Chapter 2: Big Data and Analytics for Government Innovation, pp. 23-45.

[5] J. Grimmer, "We are all social scientists now: How big data, machine learning, and causal inference work together," PS: Political Science E Politics, vol. 48, no. 01, pp. 80-83, dec 2014.

[6] Kenneth Neil Cukier and Viktor Mayer-Schoenberger, "The rise of big data," 2013.

[7] J. F. G. Vernon Turner, David Reinsel and S. Minton, "The Digital Universe of Opportunities: Rich Data and the Increasing Value of the Internet of Things," IDC Analyze the Future, IDC Analyze the Future, Tech. Rep. 1672, Apr 2014.

[8] PODS '97: Proceedings of the Sixteenth ACM SIGACT-SIGMODSIGART Symposium on Principles of Database Systems. ACM, 1997.

[9] P. Russom, "BI Search and Text Analytics: New Addition to the BI Technology Stack," TDWI Best Practices Report, The Data Warehousing Institute, Tech. Rep., Second Quarter 2007.

[10] O. Goonetilleke, T. Sellis, X. Zhang, and S. Sathe, "Twitter analytics: A big data management perspective," SIGKDD Explor. Newsl., vol. 16, no. 1, pp. 11-20, Sep. 2014.

[11] D. A. Reed and J. Dongarra, "Exascale computing and big data," Commun. ACM, vol. 58, no. 7, pp. 56-68, Jun. 2015.

[12] C. Otero and A. Peter, "Research directions for engineering big data analytics software," Intelligent Systems, IEEE, vol. 30, no. 1, pp. 13-19, Jan 2015.

[13] M. Banko and E. Brill, "Scaling to very very large corpora for natural language disambiguation," in Proceedings of the 39th Annual Meeting on Association for Computational Linguistics, ser. ACL '01. Association for Computational Linguistics, 2001, pp. 26-33.

[14] D. Dong and J. Herbert, "Record-aware compression for big textual data analysis acceleration," in Big Data (Big Data), 2015 IEEE International Conference on, Oct 2015, pp. 1183-1190.

[15] D. Jiang, B. C. Ooi, L. Shi, and S. Wu, "The performance of mapreduce: An in-depth study," Proc. VLDB Endow., vol. 3, no. 1-2, pp. 472-483, Sep 2010.

[16] R. B.-Y. Donna Harman, Edward Fox and W. Lee, Information Retrieval: Data Structures and Algorithms, Inverted files. PrenticeHall, Inc., 1992, ch. 3.

[17] P. Ferragina and G. Manzini, "Opportunistic data structures with applications," in Proceedings of the 41st Annual Symposium on Foundations of Computer Science, ser. FOCS '00. IEEE Computer Society, 2000, pp. 390-.

[18] I. H. Witten, A. Moffat, and T. C. Bell, Managing Gigabytes (2Nd Ed.): Compressing and Indexing Documents and Images. Morgan Kaufmann Publishers Inc., 1999.

[19] K. Shvachko, H. Kuang, S. Radia, and R. Chansler, "The hadoop distributed file system," in 2010 IEEE 26th Symposium on Mass Storage Systems and Technologies (MSST), May 2010, pp. 1-10.

[20] J. Dean and S. Ghemawat, "Mapreduce: simplified data processing on large clusters," Communications of the ACM, vol. 51, no. 1, pp. 107-113, 2008.

[21] D. Miner and A. Shook, MapReduce Design Patterns: Building Effective Algorithms and Analytics for Hadoop and Other Systems, 1st ed. O'Reilly Media, Inc., 2012.

[22] S. Kumar, F. Morstatter, and H. Liu, Twitter Data Analytics. Springer, 2013.

[23] J. Lin, D. Ryaboy, and K. Weil, "Full-text indexing for optimizing selection operations in large-scale data analytics," in Proceedings of the Second International Workshop on MapReduce and Its Applications, ser. MapReduce '11. ACM, 2011, pp. 59-66.

[24] J. Dittrich, J.-A. Quiané-Ruiz, A. Jindal, Y. Kargin, V. Setty, and J. Schad, "Hadoop++: Making a yellow elephant run like a cheetah (without it even noticing)," Proc. VLDB Endow., vol. 3, no. 1-2, pp. 515-529, Sep 2010. 
TABLE 4

List of public and private datasets used for the evaluation of RaPC

\begin{tabular}{l|l|r|r|l}
\hline Index & Dataset and Source & Format & Size (GB) & Description \\
\hline \hline DS-Amazon & Amazon Product Reviews [44] & TXT & 33.4 & $\begin{array}{l}\sim 35 \text { million reviews on } 2.5 \text { million products from } \sim 6.6 \text { million } \\
\text { users. }\end{array}$ \\
\hline DS-Google & Google Server Logs [43] & CSV & 158.9 & $\begin{array}{l}\sim 1.2 \text { billion server usage traces from a Google cluster (US Eastern) } \\
\text { of } \sim 11 \text { thousand machines. }\end{array}$ \\
\hline DS-Memes & Memetracker Memes [42] & TXT & 52.5 & $\begin{array}{l}\sim 96 \text { million documents; 211 million memes; and over 418 mil- } \\
\text { lion URL links from Memetracker. }\end{array}$ \\
\hline DS-PubMed & PubMed Records [40] & CSV & 3.1 & $\begin{array}{l}\sim 22 \text { million publication records of } \sim 11 \text { million authors from } \\
\text { PubMed database. }\end{array}$ \\
\hline DS-StackEX & StackExchange Posts [45] & XML & 40.6 & $\begin{array}{l}\text { An anonymized dump of user-contributed contents on the Stack } \\
\text { Exchange network. }\end{array}$ \\
\hline DS-Twitter & Tweet Topics [46] & TSV & 17.3 & $\begin{array}{l}\sim 490 \text { million tweet records. Each record consists of timesamp, } \\
\text { anonymized } \text { user_id, and topics. }\end{array}$ \\
\hline DS-Yahoo & Yahoo! Music Ratings [41] & TSV & 10.2 & $\begin{array}{l}\sim 700 \text { million ratings on } ~ 136 \text { thousand songs from } \sim 1.8 \text { million } \\
\text { users of Yahoo! Music service. }\end{array}$ \\
\hline DS-WikiEN & Wikipedia Article Abstract [47] & XML & 47.0 & The latest article abstracts (English) from Wikipedia. \\
\hline $1 G B=1,073,741,824$ Bytes
\end{tabular}

[25] S. Richter, J.-A. Quiané-Ruiz, S. Schuh, and J. Dittrich, "Towards zero-overhead static and adaptive indexing in hadoop," The VLDB Journal, vol. 23, no. 3, pp. 469-494, Jun 2014.

[26] A. Floratou, J. M. Patel, E. J. Shekita, and S. Tata, "Columnoriented storage techniques for mapreduce," Proc. VLDB Endow., vol. 4, no. 7, pp. 419-429, Apr 2011.

[27] Y. Lin, D. Agrawal, C. Chen, B. C. Ooi, and S. Wu, "Llama: Leveraging columnar storage for scalable join processing in the mapreduce framework," in Proceedings of the 2011 ACM SIGMOD International Conference on Management of Data, ser. SIGMOD '11. ACM, 2011, pp. 961-972.

[28] Y. Huai, A. Chauhan, A. Gates, G. Hagleitner, E. N. Hanson, O. O'Malley, J. Pandey, Y. Yuan, R. Lee, and X. Zhang, "Major technical advancements in apache hive," in Proceedings of the 2014 ACM SIGMOD International Conference on Management of Data, ser. SIGMOD '14. ACM, 2014, pp. 1235-1246.

[29] R. S. Rozov, Roye and E. Halperin, "Fast lossless compression via cascading bloom filters," BMC Bioinformatics, vol. 15, no. 13, p. S7, Sep 2015.

[30] H. Stranneheim, M. Käller, T. Allander, B. Andersson, L. Arvestad, and J. Lundeberg, "Classification of dna sequences using bloom filters," Bioinformatics, vol. 26, no. 13, pp. 1595-1600, 2010.

[31] B. H. Bloom, "Space/time trade-offs in hash coding with allowable errors," Commun. ACM, vol. 13, no. 7, pp. 422-426, Jul 1970.

[32] N. Asadi and J. Lin, "Fast candidate generation for real-time tweet search with bloom filter chains," ACM Trans. Inf. Syst., vol. 31, no. 3, pp. 13:1-13:36, Aug. 2013.

[33] RainStor, http://rainstor.com/, [Accessed on 20-Sep-2015].

[34] J. loup Gailly and M. Adler, http://www.gzip.org, [Accessed on 20-May-2015].

[35] D. Dong and J. Herbert, "Record-aware two-level compression for big textual data analysis acceleration," in 2015 IEEE 7th International Conference on Cloud Computing Technology and Science (CloudCom), Nov 2015, pp. 9-16.

[36] P. Deutsch, "DEFLATE Compressed Data Format Specification version 1.3," Internet Requests for Comments, RFC Editor, RFC 1951, May 1996.

[37] J. Ziv and A. Lempel, "A universal algorithm for sequential data compression," Information Theory, IEEE Transactions on, vol. 23, no. 3, pp. 337-343, May 1977.

[38] D. Huffman, "A method for the construction of minimumredundancy codes," Proceedings of the IRE, vol. 40, no. 9, pp. 10981101, Sep 1952.

[39] V. Grolmusz, "A note on the pagerank of undirected graphs," Information Processing Letters, vol. 115, no. 68, pp. 633 - 634, 2015.

[40] G. LaRowe, S. Ambre, J. Burgoon, W. Ke, and K. Brner, "The scholarly database and its utility for scientometrics research," Scientometrics, vol. 79, no. 2, pp. 219-234, 2009.

[41] Yahoo! Webscope Dataset, "dataset ydata-ymusic-user-artistratings-v1.0," http://research.yahoo.com/Academic_Relations, 2002 - 2006, [Accessed on 20-Apr-2015].
[42] J. Leskovec, L. Backstrom, and J. Kleinberg, "Meme-tracking and the dynamics of the news cycle," in Proceedings of the 15th ACM SIGKDD International Conference on Knowledge Discovery and Data Mining, ser. KDD '09. ACM, 2009, pp. 497-506.

[43] J. W. Charles Reiss and J. L. Hellerstein, "Google cluster-usage traces: format and scheme," Nov 2011.

[44] J. J. McAuley and J. Leskovec, "From amateurs to connoisseurs: Modeling the evolution of user expertise through online reviews," in Proceedings of the 22Nd International Conference on World Wide Web, ser. WWW '13. International World Wide Web Conferences Steering Committee, 2013, pp. 897-908.

[45] Internet Archive, "Stack exchange data dump," https://archive. org/details/stackexchange, [Accessed on 29-Jan-2015].

[46] L. Weng and F. Menczer, "Topicality and social impact: Diverse messages but focused messengers," arXiv, Tech. Rep., 2014.

[47] Wikimedia Database Backup EN, http://dumps.wikimedia.org/ enwiki/enwiki-latest-abstract.xml, [Accessed on 02-Dec-2014].

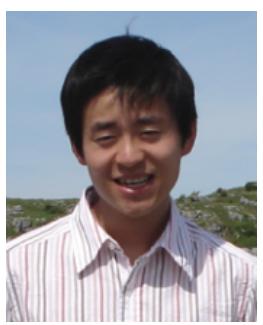

Dapeng Dong is a senior Postdoctoral Researcher at the Boole Centre for Research in Informatics of University College Cork, Ireland. He received his Ph.D. in computer science and M.Sc. in Software and Systems for Mobile Networks from University College Cork, Ireland. His research interests include self-organizing and self-managing cloud architecture, cloud resource optimization, and efficient methods for big data analytics.

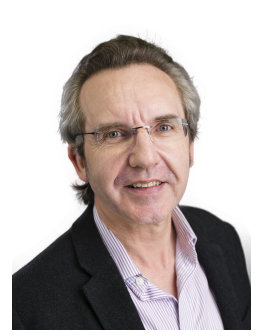

John Herbert is a senior lecturer at University College Cork, Ireland. He received his Ph.D. in computer science from the University of Cambridge, M.Sc. in Physics from University College Cork, Ireland. He has worked for SRI International, USA, Cambridge, UK, and the University of Cambridge Computer Laboratory. His research interests include modeling and implementation of architectures for pervasive computing, cloud computing, and big data analytics. 